\title{
Nft e Concerti: l'industria musicale tra liveness, sperimentazioni e alternative future
}

\author{
Alfonso Amendola \\ Università degli Studi di Salerno \\ Michelle Grillo \\ Università telematica eCampus
}

\begin{abstract}
La pandemia ha definito diverse declinazioni della liveness nella dimensione digitale. Tra i settori più colpiti e che hanno dovuto reinventare linguaggi e forme comunicative, accanto al teatro quello della musica live (il tema disciplinare sul quale vogliamo riflettere). L'industria musicale, da sempre fortemente legata alla prossimità fisica, al necessario rapporto con la strada, inteso come luogo d'ispirazione e nutrimento (immaginiamo le subculture giovanili: dai teddy boys, ai mods, dal movimento hippie, al punk, dall'hip hop alla trap) si è tenacemente reinventata negli ultimi anni negli innovativi spazi digitali: dai social network ai concerti sui videogame (sperimentando un nuovo modo di comunicare e dando vita ad un rinnovato immaginario). Attraverso questo saggio si intende procedere verso una ricognizione e analisi delle pratiche della liveness multimediali, transmediali, intermediali nell'ambito musicale esaminando, in particolare, come gli NFT musicali (Non-Fungible Tokens) si siano rivelati come una delle strade più promettenti per continuare a mantenere il legame emotivo, culturale ed identitario tra $i$ performer e i fans, e gettando le basi per un'economia alternative futura.
\end{abstract}

The coronavirus pandemic has brought about several changes in the liveness of the digital dimension. The most damaged sectors that had to rethink languages and forms of communication, include theatre but also live music which is the focus of our paper. The music industry - strictly linked to the physical proximity and to the necessary connection with the street intended as a place of inspiration and nourishment (think of youth subcultures: form Teddy Boys, to Mods, from Hippies to Punks, from Hip Hop to Trap) - has stubbornly reinvented itself in recent years making the most of digital spaces: from social networks to virtual concerts in video games (developing a new way to communicate and giving life to a renewed imagery). The aim of this paper is to move towards a survey and analysis of multimedia, transmedia, and crossmedia liveness practices in music looking in particular at how music NFTs (Non Fungible Tokens) have proved to be one of the most promising ways to preserving an emotional, cultural and identity-making connection between performers and fans. And to lay the foundations for a future alternative economy.

\section{Parolechiave/KeyWords}

Blockchain, Crypto art, Covid19, Digital sound, Performance 


\section{Introduzione}

Il 9 marzo del 2020, il Presidente del Consiglio Giuseppe Conte annunciava che la crescita del numero dei ricoverati nelle terapie intensive e delle persone decedute, richiedevano l'applicazione di norme più stringenti per affrontare il Covid19. Da quel momento l'Italia, il secondo paese più colpito dal virus dopo la Cina, si apprestava a cominciare un lungo lockdown. Mentre si cercava di comprendere la portata di quanto stesse accadendo, in nome di "io resto a casa" e "andrà tutto bene", le persone hanno fatto i conti con il distanziamento sociale e la conseguente accelerazione dei processi di digitalizzazione in molte aree della vita, dal lavoro, all'istruzione, alle relazioni sociali, allo svago. La pandemia ha infranto una serie di regole quotidiane che venivano sancite attraverso la routine. Reinventare i propri spazi e immaginarne dei nuovi, ha tendenzialmente corrisposto a una rottura definitiva con il passato. II potenziale generato ha messo in scena l'emersione non solo di nuovi sentimenti, ma anche pratiche innovative e svolte relazionali nell' ambito della scena musicale. Il lockdown causato dall'emergenza sanitaria ha generato nuovi desideri, alimentato nuove passioni e dimostrato il grande senso di creatività misto alla caratteristica sociale della resilienza che i milioni di cittadini nel mondo hanno dimostrato. La prima grande trasformazione avvenuta in tale periodo è stata, infatti, la diffusione di un elevato tasso di creatività reso possibile grazie alla disponibilità dei mezzi tecnologici ai quali poter accedere in qualsiasi momento. Molti settori hanno subito un forte contraccolpo in termini economici, i più colpiti sono stati quelli dell'arte e della cultura in generale. Il teatro è stato investito da un'importante caduta, colpito sia nel pieno della stagione invernale 2019-2020 con la brusca interruzione avvenuta a partire dalla seconda metà di febbraio in regioni traino per il settore come la Lombardia e I'Emilia Romagna, e poi a seguire nel resto d'Italia, sia ad avvio della stagione 2020-2021 con il secondo lockdown. Stesso dicasi per l'attività concertistica; quest'ultima, in particolare, è quella che più ha risentito, in valori assoluti, della pandemia; con una perdita dell' $83 \%$ degli spettatori e un crollo della spesa al botteghino pari 
all'89\%. Le capienze ridotte non hanno consentito neanche in estate l'allestimento di eventi dei grandi artisti nazionali e internazionali ${ }^{1}$.

Stesso discorso per quanto riguarda la moda che nel 2020 ha dovuto rinunciare alla dimensione fisica della strada e della passerella, per trovare nuovi posizionamenti in spazi più o meno inediti allo scopo di mantenere un rapporto di continuità con il proprio pubblico e cogliere, al contempo, l'opportunità di intercettarne di nuovi. La moda ha esplorato la ricchezza dello spazio virtuale dei videogame, dove le Maison, in nome del "game-bait", sono approdate. Insieme alla moda, con una certa continuità che da sempre le unisce, anche la musica è approdata nei videogame. Diventando lo spazio perfetto per la realizzazione di nuove e originali forme di concerti. II 4 aprile 2020 - in pieno lockdown - il rapper Travis Scott ha radunato 12milioni di utenti sulla piattaforma Fortnite per presentare il nuovo singolo The Scotts. L'evento è poi stato replicato sulla piattaforma quel tanto da essere definito Tour, secondo una logica transmediale che vede al centro il progetto musicale, ma con un chiaro interno di posizionamento dell'artista in una logica di cross marketing. A tal riguardo Brembilla fa notare che le strategie transmediali nell'industria musicale possono essere diverse e possono combinare vecchi e nuovi modelli ${ }^{2}$. Il concerto live di Travis Scott è probabilmente l'esempio più rappresentativo dell'evoluzione in seguito alla pandemia. I concerti dal vivo, così come il teatro, hanno dovuto reinventare un nuovo linguaggio e forme comunicative trovando posto in spazi inesplorati, oppure andando a intensificare forme ed esperienze già in essere, come nel caso dei Couch Tour e delle live su YouTube, Instagram e Facebook, e in altri casi, dando vita a modalità assolutamente inedite di fruizione, come i concerti in NFT.

\section{La liveness dell'industria musicale}

In seguito alla pandemia si è molto riflettuto sul concetto di liveness e sugli effetti del Covid 19 sulle esperienze live che vivono sostanzialmente della prossimità fisica, mettendo in discussione il valore del qui e ora. Proprio lungo la lineare del "qui ed ora" si sono svilup-

\footnotetext{
${ }^{1}$ Annuario dello spettacolo 2020 https://www.siae.it/sites/default/files/SIAE_Annuario_dello_Spettacolo_2020.pdf (ultimo accesso 10/01/2022).

${ }^{2} \mathrm{P}$. Brembilla, Transmedia Music: The Values of Music as a Transmedia Asset, in M. Freeman, R. Rampazzo Gambarato (a cura di), The Routledge Companion to Transmedia Studies, Routledge, New York 2019, pp. 82-89.
} 
pate diverse produzioni. Un progetto di ricerca realizzato presso il Massachusetts Institute of Technology (MIT) ha trasformato il coronavirus in una melodia. Per farlo, un team di ricercatori ha utilizzato un'intelligenza artificiale e la tecnica che quest'ultima possiede di tradurre in musica il Covid-19. L'intelligenza artificiale ha trasposto in suoni e vibrazioni le sequenze di amminoacidi che compongono la catena proteica del virus. La melodia è di ben 109 minuti ed è possibile ascoltarla sul Soundcloud del professore Markus Buehler.

Se da un lato la musica si è fatta portatrice di capacità scientifiche come nel caso prima descritto, si sono verificate eventualità in cui la musica si è posta al servizio della salvaguardia del benessere psicologico di un individuo o di un insieme di essi. Divenuta la vera protagonista durante il lockdown, la musica ha conferito sollievo e profonda emozione, con scenari toccanti e inimmaginabili. Grazie all'utilizzo dei social network è stato possibile creare una rete di contatti per darsi appuntamento a una data ora per dare vita a una vera e propria performance estemporanea. I balconi e le finestre delle abitazioni sono diventati palcoscenici per rappresentazioni musicali e spettacoli spontanei, in un sostanziale e radicale ripensamento del concetto di liveness. Le prime riflessioni sulla liveness le dobbiamo a Philip Auslander, incentrate in modo particolare sulla performance, l'arte e la relazione con i media e la tecnologia; con uno sguardo particolare al teatro, la recitazione cinematografica, la performance art, il cabaret e la musica. Nel suo lavoro riguardante il rapporto tra l'esecuzione live e quella "medializzata", Auslander smonta l'idea di un'opposizione tra le due, ma stabilisce un processo di ridefinizione reciproca. "The relationship between live performance and its present mediatized environment, they yield a reductive binary opposition of the live and the mediatized $»^{3}$. Questo approccio torna ancora particolarmente utile alla luce dell'immersione online di questi ultimi mesi.

\footnotetext{
Whereas the traditional view represented (...) sees theatre and the live performance arts generally as belonging to a cultural system separate from that of the mass media, live forms have become mediatized in Jameson's sense: they have been forced by economic reality to acknowledge their status as media within a mediatic system that includes the mass media and information technologies. Implicitly acknowledging this situation, a number of theatres have displayed signs similar to the banner that flew outside the Alliance Theater in Atlanta declaring that its offerings are "Not Available on Video," demonstrating that the only way of
}

${ }^{3}$ P. Auslander, Liveness: Performance in a Mediatized Culture, Routledge, London and New York 2008, p. 15. 
imputing specificity to the experience of live performance in the current cultural climate is by reference to the dominant experience of mediatization. ${ }^{4}$

Partendo infatti dal principio che il live in sé non è uno stato incontaminato dai media ma, anzi, nasce insieme alla tecnologica, vediamo come la storia della performance dal vivo è legata indissolubilmente alla storia dei supporti di registrazione e si estende per non più degli ultimi 100-150 anni. «Lungi dall'essere invasa, contaminata o minacciata dalla mediazione, la performance dal vivo è sempre già inserita con le tracce della possibilità di mediazione tecnica che la definisce come dal vivo ${ }^{5}$. In particolare, Auslander, affrontando quella che è la performance musicale, assume una posizione abbastanza forte sulla distinzione tra la musica come essenza e l'essere fisico di chi la fa, in particolare attraverso i suoi studi sulle performance di David Bowie. Egli afferma: «la musica non è un suono disgiunto dall'essere fisico di chi lo fa [...] i suoni che sento risultano direttamente da tutti gli aspetti dell'impegno fisico della persona e dell'atto di fare musica - tutti i suoni e i gesti che costituiscono la performance ${ }^{6}$. Tale affermazione rappresenta il risultato e la naturale evoluzione di un'indagine volta a mettere in discussione l'idea ancora diffusa che in una performance musicale sia utile o possibile ancora distinguere la musica dalla tecnica.

Live performance now often incorporates mediatization to the degree that the live event itself is a product of media technologies. This has been the case to some degree for a long time, of course: as soon as electric amplification is used, one might say that an event is mediatized. What we actually hear is the vibration of a speaker, a reproduction by technological means of a sound picked up by a microphone, not the original (live) acoustic event. Recently, however, this effect has been intensified across a very wide range of performance genres and cultural contexts, from the giant television screens at sports arenas to the video apparatus used in much performance art. The spectator sitting in the back rows of a Rolling Stones or Bruce Springsteen concert, or even a Bill Cosby stand-up comedy performance, is present at a live performance, but hardly participates in it as such since his/her main experience of the performance is to read it off a video monitor. ${ }^{7}$

\footnotetext{
${ }^{4}$ P. Auslander, op. cit., p. 18.

5 P. Auslander, Digital Liveness: Historico-Philosophical Perspective, in «PAJ: A Journal of Performance and Art», vol. 34, n. 3, 2012, pp. 3-11 [traduzione nostra].

${ }^{6}$ P. Auslander, Music as Performance: The Disciplinary Dilemma Revisited, in N. Cook, R. Pettengill (a cura di), Taking it to the Bridge: Music as Performance, University of Michigan Press, Ann Arbor 2013, pp. 249- 357 [traduzione nostra].

${ }^{7}$ P. Auslander, Liveness: Performance in a Mediatized Culture, Routledge, London and New York 2008, p. 40.
} 
Auslander pone poi la questione sul pubblico e cioè tra quello che assiste allo show originale e quello che ne osserva la riproduzione, secondo cui l'autenticità deriverebbe dal percepire la performance riprodotta come facente parte del progetto estetico dell'artista. Lo stesso concerto live, di cui conserviamo ingenuamente un'idea di purezza, che cos'è se non uno spettacolo in serie mediato sempre e comunque dalla tecnologia e i software audio, i giochi di luce, i mega schermi e tutti gli effetti speciali, fanno parte del grande evento medializzato, anche se il performer deve apparire vero, e il concerto percepito come unico e irripetibile.

\section{Rivoluzione o evoluzione: Convergenza e transmedialità dell'evento live.}

Con la pandemia abbiamo assistito alla più alta espressione di convergenza secondo la definizione di Jenkins: «il flusso di contenuti su più piattaforme, la cooperazione tra più settori dell'industria dei media e il migrare del pubblico alla ricerca continua di nuove esperienze di intrattenimento ${ }^{8}$. Complessivamente gli eventi live sono diminuiti di quasi il $70 \%$, gli ingressi del 73\%. La spesa al botteghino è scesa del 78\%, con un $-92,04 \%$ solo per quel che riguarda il settore dei concerti di musica leggera, che in termini assoluti vale 355,5 milioni ${ }^{9}$. Mentre gli eventi live venivano annullati o nella migliore delle ipotesi rimandati a data da destinarsi, molti venivano eseguiti in streaming. La vendita di biglietti online ha segnato un $+292 \%$ tra giugno e dicembre 2020 secondo MIDia report ${ }^{10}$, tanto che dal 2020 i dati sul macro aggregato degli eventi streaming su piattaforme digitali sono strati inseriti nell'annuario dello spettacolo redatto dalla SIAE. Si è assistito a un incremento delle dirette Facebook e Instagram. Un esempio sono state le live casalinghe per invitare le persone a non uscire di casa. Usando I'hashtag \#Togetherathome il 16 marzo 2020 Chris Martin dei Coldplay ha tenuto uno speciale concerto da casa, live su Instagram, uno show di circa trenta minuti nel quale ha eseguito A Sky full of stars; Trouble, Viva la Vida. Il giorno successivo anche il cantante John Legend ha eseguito il suo concerto live streaming. Dalla performance di

\footnotetext{
8 Jenkins H., Cultura Convergente, Apogeo, Milano 2007, p. XXV.

${ }^{9}$ Annuario dello spettacolo 2020:

https://www.siae.it/sites/default/files/SIAE_Annuario_dello_Spettacolo_2020.pdf (ultimo accesso 10/01/2022).

${ }^{10}$ A. Zo, Blockchain e Nft per un'industria musicale più sostenibile (e libera), 04/03/2021:

https://www.wired.it/play/musica/2021/03/04/blockchain-nfts-industria-musicale-sostenibile (ultimo accesso 10/01/2022).
} 
Chris Martin su Instagram è nato il progetto One World: Togetherat Home, l'evento più grande in epoca Covid-19, un concerto virtuale in diretta mondiale per raccogliere fondi a favore dell'Organizzazione Mondiale della Sanità e in particolare del fondo per le iniziative di contrasto alla pandemia chiamato Solidarity Response Fund. Un'iniziativa di Global Citizen il cui programma è stato curato da Lady Gaga. Quarantacinque performance digitali in un diretta transmediale su TV e piattaforme video, otto ore e quasi ottanta artisti che si sono esibiti in due segmenti: il primo digitale della durata di sei ore, il secondo segmento televisivo presentato da Jimmy Fallon, Jimmy Kimmel e Stephen Colbert è stato trasmesso in mezzo mondo e in Italia è andato in onda su Rai 1 e su Rai Radio 2 a partire dalle ore 1:45, con la conduzione di Ema Stokholma e Fabio Canino, evento replicato poi su Rai Radio 2, su MTV (e in streaming su Now TV) e Comedy Central. Tra gli artisti che hanno preso parte all'evento: Adam Lambert, Annie Lennox, Ben Platt, Cassper Nyovest, Christine And The Queens, Common, Delta Goodrem, Finneas, Jack Johnson, Jacky Cheung, Jess Glynne, Jessie J, Juanes, Kesha, Michael Bublé, Rita Ora, Sebastián Yatra, Sheryl Crow, Sho Madjozi, Sofi Tukker, The Killers, Zucchero, Angèle, Billy RayCyrus, Charlie Puth, Eason Chan, Ellie Goulding, Hozier, Jennifer Hudson, John Legend, Lady Antebellum, Leslie Odom Jr., Luis Fonsi, Niall Horan, Picture This, Super M, Alicia Keys, Andrea Bocelli, Billie Eilish, Billie Joe Armstrong, Camila Cabello, Celine Dion, Chris Martin, Eddie Vedder, Elton John, J Balvin, Jennifer Lopez, Lady Gaga, Lizzo, LL Cool J, Paul McCartney, Pharrell Williams, Rolling Stones, Sam Smith, Shawn Mendes, Stevie Wonder, Taylor Swift e Usher ${ }^{11}$.

Si potrebbe affermare che la pandemia abbia messo in moto la piena rivoluzione digitale nel mondo della musica live? Certo è che nel tempo ferito della pandemia il potenziale cognitivo umano è venuto a galla. In questo momento di profonda crisi è esploso come una dimostrazione di volontà e necessità di rimanere in contatto nonostante la distanza. L'ipertropia degli schermi e del flusso di informazioni attraverso di essi veicolate, rischiava di rendere atrofizzato l'uomo. L'ipnosi alla quale sembrava poter essere soggetto è divenuta una forma di estensione delle proprie capacità psico-fisiche.

\footnotetext{
11 Rolling Stone, Guarda l'evento One World: TogetherAtHome, 18/04/20202: https://www.rollingstone.it/musica/guarda-levento-one-world-together-at-home/513109 (ultimo accesso 10/01/2022).
} 
Un'immagine in televisione trasmessa durante il periodo di quarantena diveniva concretizzazione di sentimenti comuni. Basti pensare alle numerose pubblicità sviluppate durante tutto il periodo del lockdown: il cambiamento dei registri linguistici, la messa in scena di città vuote, l'indossare la mascherina e la volontà di ricominciare sono divenuti le iconografie per immagini e per sensi, per eccellenza, simboli di una riscoperta umanità. Gli schermi non possono essere considerati, però, solo come dei fornitori di immagini. Oggi i display sono bacheche che danno la possibilità di scegliere all'utenza cosa, quando, come e dove cliccare. Possono essere considerati specchi della propria persona "digitalizzata", come i diari sui social network, o ancora, finestre dalle quali affacciarsi sul mondo. È fondamentale, perciò, tenere a mente che uno schermo, o per meglio dire, un display, non ha potere manipolativo sull'uomo, ma è quest'ultimo che lo possiede e lo modella a proprio piacimento.

Sicuramente la social media society, dal canto suo, con strumenti potentissimi quale il largo raggio di trasmissione di una informazione, ha sempre viaggiato sul filo del rasoio a metà tra potenziale divulgativo e disinformazione dovuta all'emissione di fake news non controllate in partenza, ma a contribuire alla diffusione di informazioni vi era la produzione personale ed individuale su ogni singolo "diario" sociale.

Le bacheche dei maggiori social network si riempivano giornalmente di informazioni relative alla propria sfera quotidiana, al modo in cui venivano riadattate le abitudini, a come si stesse svolgendo la rilocazione alla quale ognuno era costretto.

Tali processi sociologico-mediali potrebbero essere la piena realizzazione di una teoria promossa da Henry Jenkins. A lui, nel 2006, va il merito di aver perfezionato in un testo di importanza epocale, quella che è la teoria della "cultura della convergenza", accennata in precedenza, secondo la quale una narrazione transmediale corrisponde ad una forma narrativa che muovendosi attraverso più strutture e forme mediali può divenire perfezionamento dell'esperienza del prosumer e di nuove e distinte forme di informazione. La produzione di contenuti digitali con tecniche "dal basso" proprie dei "non addetti ai lavori" diventano, dunque, vere forme artistiche di cui ci si serve per ricostruire un dato momento o periodo passato.

Le testimonianze musicali e di liveness di coloro i quali hanno ripensato il proprio impegno creativo e visionario durante la pandemia, raccontate attraverso la social media 
society o il sistema media-audiovisivo, hanno evidenziato le capacità di un individuo di farsi produttore e creatore di tali contenuti digitali che siano in possesso di una storia e che siano costruiti esteticamente performativi, con un contenuto coerente e coeso.

Sarebbe importante notare come un fattore esterno, lontano dalla capacità di manipolazione dell'uomo e di portata smisuratamente preoccupante, quale può essere la diffusione di un virus, sia fonte di creatività e inneschi nelle menti una volontà di manifestare il proprio pensiero attraverso ogni possibile supporto mediale (ma questo apre ad altri spazi di riflessione).

In ogni caso la Pandemia ha fatto ripensare per intero il processo digitale; e invita a ragionare sull'idea stessa di rivoluzione digitale, un'idea forse abusata che presuppone un prima e un dopo, e un vecchio e un nuovo. Rispetto all'idea del voler spazzare via il primo in favore del nuovo, Pasquali ricorda: «La separazione fra nuovi e vecchi media è, più che un dato di fatto, un effetto delle distorsioni prospettiche operate dalla retorica del nuovo che tende ad appiattire la complessità dei vecchi media» ${ }^{12}$.

È indubbio che le nuove tecnologie digitali abbiano cambiato il modo di produrre e consumare la musica, ma più che parlare di rivoluzione, come fa notare Gianni Sibilla, sarebbe più opportuno parlare di evoluzione digitale ${ }^{13}$. Anzi è evidente che supporti, organizzazione dei linguaggi, accessi e produzioni, spesso riprendono stili e modalità dei vecchi media, un po' perché i nuovi media ne sono la continuazione ${ }^{14}$. E in effetti già McLuhan affermava che ogni nuovo mezzo di comunicazione tende a riassumere in sé molte delle funzioni assolte dai mezzi precedenti ${ }^{15}$. In particolare, nel campo della musica, come nota Sibilla, non c'è mai stata una vera e propria rottura, ma il mutamento in atto è stato costante dall'inizio del XX secolo con importanti accelerazioni negli ultimi anni, ma difatti non c'è mai stata una vera e propria rottura, ma piuttosto sostituzioni graduali, ciò che è cambiata è la velocità di tali sostituzioni. Secondo Sibilla i nuovi media non hanno fatto altro che trasferire in uno spazio nuovo il sistema dei media musicali, dischi che sono diventati MP3, performance trasmesse in live streaming, i discorsi dei fan trasferiti

\footnotetext{
${ }^{12}$ F. Pasquali, I nuovi media. Tecnologie e discorsi sociali, Carocci, Roma 2003, p.29.

${ }^{13} \mathrm{G}$. Sibilla, Musica e media digitali. Tecnologie, linguaggi e forme sociali dei suoni, dal walkman all'iPod, Bompiani, Milano 2008.

${ }^{14}$ R. Stella, C. Riva, C. M. Scarcelli, M. Drusian, Sociologia dei new media, Utet, Torino 2018.

${ }^{15}$ M. McLuhan, Gli strumenti del comunicare, Il Saggiatore, Milano 1990.
} 
nelle chat e nelle community sui social network. Una non rivoluzione che secondo Bolter e Grusin sarebbe tipica dei media stessi, la cui caratteristica fondamentale è la rielaborazione delle forme comunicative precedenti attraverso il processo di rimediazione ${ }^{16}$. ̀̀ questo il caso dei Couch Tour che rappresentano una modalità produttiva e di consumo consolidata da anni, in cui i fan si riuniscono online per assistere a spettacoli in tempo reale, e grazie ai quali, in piena pandemia, il settore della musica live, ha trovato il modo di esistere al di fuori dei luoghi fisici. Il fenomeno dei Couch Tour è già presente da un decennio, i social network rappresentano un'ulteriore evoluzione che hanno reso attiva e partecipativa la trasmissione Couch Tour, il cui hub è Nugs.TV, lanciato nel 1993 come sito per fan dove condividere audio gratuiti. Oggi Nugs.Tv offre una consistente lista di spettacoli insieme a importanti archivi che includono concerti di Bruce Springsteen, Pearl Jam, Sonic Youth, Metallica. Questi ultimi, consapevoli della forza aggregativa del web, hanno tenuto il primo concerto completo in streaming: era il 1995. A parte poche e visionarie band, fino a qualche anno fa i musicisti erano particolarmente scettici riguardo le performance realizzate su piattaforme video o per il web, si è visto con Auslander come nei suoi studi tale dicotomia sia stata annullata. Da qualche anno assistiamo a una crescita dei format live sulle piattaforme social, ma anche su Netflix, Prime Video, The National nelle quali i contenuti vengono di volta in volta declinati secondo ogni formato, cambiando linguaggi e regole.

\section{Dai concerti live streaming agli NFT}

Rispetto a una ripresa che procede sotto il segno della prudenza, Boccia Altieri e Laura Gemini ipotizzano per il futuro forme blended di spettacolo dal vivo ${ }^{17}$. L'accesso illimitato ai contenuti sembra spazzare via d'un colpo concetti come "unicità" e "esclusività". Non si può non pensare alle teorizzazioni di Walter Benjamin che all'inizio del secolo scorso si interrogava sulla perdita dell'aura a causa della riproducibilità tecnica

\footnotetext{
${ }^{16}$ J.D. Bolter, R. Grusin, Remediation: competizione e integrazione tra media vecchi e nuovi, Guerini e Associati, Milano 2002.

${ }^{17} \mathrm{G}$. Boccia Artieri, L. Gemini, Per un live dei corpi a distanza, in "Doppiozero", $1^{\circ}$ maggio 2020, https://www.doppiozero.com/materiali/un-live-dei-corpi-distanza (ultimo accesso 10/01/2022).
} 
La tecnica di riproduzione, moltiplicando la riproduzione, pone al posto di un evento unico una sua grande quantità. [...] Nell'istante, pero, in cui alla produzione manca il criterio dell'autenticità dell'arte, anche l'intera funzione sociale dell'arte si trasforma. Al posto di una sua fondazione nel rituale s'instaura una fondazione su una prassi diversa, vale a dire un suo fondarsi sulla politica. ${ }^{18}$

Intorno alle performance online, ha preso piede un altro format, quello degli NFT (nonfungible token) che offrono una risposta al problema della proprietà del bene virtuale. Gli NFT sono una tecnologia in grado di trasformare i file in entità digitali uniche grazie all'utilizzo della blockchain, ovvero un registro digitale cronologico non modificabile all'interno del quale ogni transazione, una volta scritta, non si può modificare, né cancellare, garantendone la proprietà e l'unicità. Ciò che cambia è che le opere, in quanto digitali rimangono fruibili, ma la proprietà resta esclusiva. II settore, secondo DappRadar, società che analizza applicazioni decentralizzate, comprese le piattaforme di vendita degli NFT basate su blockchain, il fenomeno è esploso negli ultimi 12 mesi e solo nella prima metà del 2021 ha prodotto un volume di vendite stimato nell'ordine dei 2,5 miliardi di dollari. Nello stesso periodo dell'anno precedente il valore era di 13,7 milioni di dollari ${ }^{19}$. Se il settore dell'arte è stato precursore degli NFT, oggi quello della musica rappresenta un nuovo approdo.

Kenneth Chi Ho Kim, nei suoi studi sull'impatto delle blockchain sull'industria musicale, ne spiega il funzionamento:

\begin{abstract}
When the music is purchased by the customer, the transaction is recorded on the new block which is created and shared among stakeholders of the blockchain. After stakeholders approve the transaction, the new block added to the chain of blocks and the payment is automatically sent to the right holder. The means of the payment for the blockchain music is cryptocurrency such as Bitcoin and Ethereum. In the era of digital technology, the aggregator was added to the recorded music supply chain to deliver digital music to consumers. ${ }^{20}$
\end{abstract}

Facilmente scambiabili e individualmente unici, si trovano su determinati blockchain: Ethereum, Flow, Wax, Bitcoin, Algorand. Rispetto alle cryptovalute gli NFT sono completamente e non direttamente intercambiabili con qualsiasi altra risorsa. Ogni fungible token

\footnotetext{
${ }^{18}$ W. Benjamin, L'opera d'arte all'epoca della sua riproducibilità tecnica, Einaudi, Torino 2014.

19 HDBlog, Con gli NFT si fanno miliardi di dollari: 2,5 nella prima metà dell'anno, 7/07/2021: https://www.hdblog.it/mobile/articoli/n540833/nft-mercato-miliardi-2021-analisi/ (ultimo accesso 10/01/2022).

${ }^{20}$ K.C.H. Kim, The Impact of Blockchain Technology on the Music Industry in «International Journal of Advanced Smart Convergence», 2019, vol. 8, n.1, pp. 196-203.
} 
è composto da metadati che ne conferiscono l'individualità. Gli NFT non esistono se non nello spazio virtuale ma possono essere applicati a ogni prodotto fisico, diventando il gemello digitale, una soluzione contro la contraffazione dei prodotti. La creazione di un NFT per ogni prodotto reale crea soprattutto un ponte che unisce il mondo reale al Metaverso, dove per Metaverso non intendiamo il mondo digitale, ma un mondo parallelo, un luogo dove in futuro sarà possibile trascorrere un tempo da destinare allo svago, alle relazioni sociali e all'acquisto e il consumo di prodotti. È evidente che gli Nft creano già da oggi i presupposti per una relazione tra un brand e chi abiterà il Metaverso, consentendo la creazione del valore economico degli oggetti presenti al suo interno. Se nella vita fisica, abbiamo la proprietà dei beni: auto, abiti, case, lo stesso può avvenire nel Metaverso in cui ogni singolo asset può essere acquistato, venduto o scambiato attraverso gli Nft.

Gli Nft possono essere quindi qualsiasi contenuto digitale - video, meme, musica - e potranno essere utilizzati in modo inedito su giochi online e siti internet e poiché ne decretano l'unicità e la proprietà rappresentano un'opportunità per le industrie creative. I Kings of Leon sono entrati nel mercato degli NFT con il nuovo album When you see yourself ${ }^{21}$, pubblicato su Amazon Music, Spotify, Apple Music, è stato pubblicato anche in NFT sulla piattaforma musicale YellowHeart.com con tre diversi token di cui uno includeva vantaggi per gli spettacoli dal vivo. La YellowHeart ha poi coniato 18 biglietti d'oro che hanno sboccato i biglietti reali per i concerti in prima fila. Inoltre, per aumentarne il valore, la band ha messo all'asta sei biglietti NFT, gli altri dodici sono stati emessi uno alla volta proprio come delle opere d'arte. Il parallelismo non è casuale perché il valore è definito non tanto dal contenuto che viene incorporato, ma dalla prova di autenticità che viene direttamente dall'artista. L'importanza degli NFT deriva appunto, dall'evoluzione nel rapporto tra l'artista e la propria base fan, un'evoluzione resa possibile già grazie alle fandom e prima ancora alle fanzine. In particolare, risolve economicamente il sistema delle piattaforme streaming che rappresenta oggi i quattro quinti del mercato musicale e dal quale l'autore realizza un guadagno minimo, i veri introiti sono quelli degli spettacoli dal vivo che nell'ultimo anno e mezzo sono venuti a mancare. Gli NFT garantiscono un volume d'affari e opportunità economiche più elevate,

\footnotetext{
${ }^{21}$ S. Hisson, I Kings of Leon saranno la prima band a pubblicare un album come NFT 3/03/2021, https://www.rollingstone.com/pro/news/kings-of-leon-when-you-see-yourself-album-nft-crypto-1135192/
} 
dando agli ascoltatori/fruitori del prodotto la piena proprietà, l'artista conserva il diritto di autore e il diritto di riproduzione ma in ogni caso la proprietà originale resta in possesso dell'acquirente. Un attaccamento, quello all'originale, che rimane anche di fronte alla smaterializzazione dei beni, deducibile sicuramente dall'incremento del mercato del vinile nel 2021 ( i dati di Deloitte per FIMI mostrano una crescita complessiva del mercato discografico del $34 \%$ e una crescita del mercato del vinile del $189 \%$, scavalcando il CD) ${ }^{22}$. Gli NFT rappresenterebbero, in senso più ampio, un elemento per non perdere di vista l'attaccamento ai beni, intesi come prodotti, per quanto essi siano immateriali, il presupposto necessario per creare un mercato tangibile nel futuro Metaverso. La traccia per legittimare in futuro la struttura del Metaverso, influenzandone la sovrastruttura ${ }^{23}$, dal momento che stiamo esperendo l'inversione della gerarchia e dell'equilibrio tra l'immateriale e il materiale in modo completamente inatteso a favore del primo elemento ${ }^{24}$. In senso più stretto, oggi, l'applicazione degli NFT in campo musicale equilibrano il mercato musicale spezzando la catena del modello dei contenuti spesso gratuiti, analizzati da Chris Anderson nel $2009^{25}$. Anderson aveva individuato una nuova economia basata sullo sviluppo della freeconomic, in cui una generazione cresciuta con Google, dava, e dà ancora per scontato che ogni bene digitale sia gratis, interiorizzando le sottili dinamiche del marketing del costo zero. I modelli di business più interessanti nel primo decennio del duemila consistevano, infatti, nell'individuazione di un guadagno attraverso il concetto del free; le linee di tendenza delle attività economiche online di allora puntavano tutte nella stessa direzione: verso la gratuita dei contenuti. La cui fruizione dei beni materiali, che fino a un decennio prima era centrata sul diritto di proprietà dei beni reali, era stata sostituita dai contenuti digitali la cui economia basata su replicabilità e illimitatezza, sembravano aver spazzato via d'un colpo concetti come "unicità" e "esclusività" dei beni. Oggi è evidente che si sta procedendo verso la direzione opposta, un modello che non mette in crisi lo sharing, ma che ne definisce con chiarezza la proprietà. Gli NFT risolvono in parte la questione dell'incontrollabilità dei file e della digitalizzazione garantendo la tracciabilità dei processi.

\footnotetext{
${ }^{22}$ Mercato italiano in crescita del 34\% nel primo semestre 2021, https://www.fimi.it/mercato-musicale/dati-dimercato/mercato-italiano-in-crescita-del-34-nel-primo-semestre-2021.kl

${ }^{23}$ K. Marx, Per la critica dell'economia politica [1859], Clinamen, Firenze 2011.

${ }^{24}$ C. Attimonelli, V. Susca, Un oscuro riflettere. Black Mirror e l'aurora digitale, Mimesis, Milano, 2020.

${ }^{25}$ C. Anderson, Gratis, Rizzoli, Milano 2010.
} 
In futuro è ora? Molto probabilmente sì. I recenti dati sulle vendite indicano che nel marzo del 2021, il DJ e produttore musicale 3LAV ha venduto NFT musicali per 11,6 miliardi di dollari in sole 24 ore. La musicista canadese Grimes ha guadagnato 5,8 miliardi in soli venti minuti. Rispetto all'abbondanza dei flussi digitali, gli NFT rendono possibile la scarsità digitale, un esempio è dato dall'operazione realizzata da Rac, pseudonimo di André Allen Anjosche che nel maggio 2020 ha rilasciato il suo ultimo album in digitale e in musicassette. Un'operazione in chiave nostalgia e al contempo esclusiva: solo cento musicassette, rappresentate da un NFT chiamato Tape, e ogni token dal valore di 28 dollari, il prezzo è poi arrivato a 13 mila dollari l'uno, la cifra più alta mai spesa per una musicassetta. Oggi le principali piattaforme per vendere musica in NFT sono SuperRare, Nifty Gateway e Blockparty. Nifty Gateway, rispetto alle altre è una piattaforma crypto che consente pagamenti con carta di credito e non solo con cryptovalute. Intanto in Italia anche la Siae procede verso la realizzazione di una piattaforma blockchain per tracciare in modo trasparente la gestione del diritto d'autore e i pagamenti, mentre artisti vanno alla conquista del nuovo mercato, tra questi Morgan. Il duo romano dei Belladonna si è reso protagonista di un'operazione di vendita di NFT molto particolare. Comprendeva non solo l'inedito in copia unica digitale New Future Travelogue, ma anche i suoi diritti di copyright su eventuali utilizzi futuri. Davide Dileo, in arte Boosta, il tastierista dei Subsonica e il cantautore e scrittore croato Danijel Žeželj, con la startup Genuino, hanno lanciato il progetto Music is art sulla piattaforma Makersplace, una collezione di NFT fatta di composizioni musicali e Visual Art. Chi ha acquistato i contenuti ha avuto anche la possibilità di assistere in forma esclusiva a una loro performance dal vivo, un vero e proprio NFT live che unisce l'offline e l'online. NTF che uniscono anche passato e presente della musica, la cantante Whitney Houston avrà il suo lavoro in NFT, si tratterà di una canzone inedita registrata all'età di 17 anni e sarà venduta durante la Miami Art Week del $1^{\circ}$ dicembre $2021^{26}$.

Esclusività e unione tra online e offline sono le leve degli Nft che si realizzano attraverso l'esperienza della biglietteria evento dal vivo, non solo i fan possono accedere a contenuti digitali esclusivi acquistando e detenendo NFT di un artista, il fan può ricevere

\footnotetext{
${ }^{26}$ S. Grant, One of announces Whitney Houston NFT collection, in «The Source», $19^{\text {th }}$ november 2021, https://thesource.com/2021/11/18/oneof-announces-whitney-houston-nft-collection/
} 
un trattamento o un accesso speciale, sconti, offerte o contenuti aggiuntivo specifico dell'evento in base ai token in loro possesso, inoltre, attraverso la tecnologia NFT è possibile tenere un registro immutabile sulla blockchain, certificando che quel singolo fan era in quello specifico spettacolo. Le possibilità sono infinite e siamo solo all'inizio.

\section{Riferimenti Bibliografici}

C. Anderson, Gratis, Rizzoli, Milano 2010.

C. Attimonelli, V. Susca, Un oscuro riflettere. Black Mirror e l'aurora digitale, Mimesis, Milano 2020.

P. Auslander, Liveness. Performance in mediatized culture, Routledge, London-New York, 1999.

P. Auslander, Performing Glam Rock. Gender and Theatricality in Popular Music, The University of Michigan Press, Ann Arbor 2006.

P. Auslander, Music as Performance: The Disciplinary Dilemma Revisited, in N. Cook, R. Pettengill (a cura di), Taking into the Bridge: Music as Performance, University of Michigan Press, Ann Arbor 2013.

W. Benjamin, L'opera d'arte all'epoca della riproducibilità tecnica [1936], Einaudi, Torino 2000.

G. Boccia Altieri, L. Gemini, Per un live dei corpi a distanza, in «Doppiozero», $1^{\circ}$ maggio 2020, https://www.doppiozero.com/materiali/un-live-dei-corpi-distanza

J.D. Bolter,R. Grusin, Remediation: competizione e integrazione tra media vecchi e nuovi, Guerini e Associati, Milano 2002.

P. Brembilla, Transmedia Music: The Values of Music as a Transmedia Asset, in M. Freeman, R. Rampazzo Gambarato (a cura di), The Routledge Companion to Transmedia Studies, Routledge, New York 2019, pp. 82-89.

L. Gemini, Liveness: le logiche mediali nella comunicazione dal vivo, in «Sociologia della Comunicazione», n. 51, 2016, pp. 43-63.

L. Gemini, S. Brilli, Il pubblico del teatro contemporaneo: fra auto-osservazione e processi di fruizione, in «Comunicazioni sociali», n. 3, 2018, pp. 448-462.

H. Jenkins, Cultura Convergente, Apogeo, Milano 2007.

K. Marx, Per la critica dell'economia politica [1859], Clinamen, Firenze 2011.

M. McLuhan, Gli strumenti del comunicare, II Saggiatore, Milano 1990.

F. Pasquali, I nuovi media. Tecnologie e discorsi sociali, Carocci, Roma 2003. 
G. Sibilla, Musica e media digitali. Tecnologie, linguaggi e forme sociali dei suoni, dal walkman all'iPod, Bompiani, Milano 2008.

R. Stella, C. Riva, C. M. Scarcelli, M. Drusian, Sociologia dei new media, Utet, Torino 2018.

\section{Sitografia}

https://www.hdblog.it/mobile/articoli/n540833/nft-mercato-miliardi-2021-analisi (ultimo accesso il 3/11/2021)

https://www.rollingstone.com/pro/news/kings-of-leon-when-you-see-yourself-album-nftcrypto-1135192 (ultimo accesso 3/11/2021)

https://www.fimi.it/mercato-musicale/dati-di-mercato/mercato-italiano-in-crescita-del-34nel-primo-semestre-2021.kl (ultimo accesso 5/11/2021)

https://www.siae.it/sites/default/files/SIAE_Annuario_dello_Spettacolo_2020.pdf (ultimo accesso 10/01/2022)

https://thesource.com/2021/11/18/oneof-announces-whitney-houston-nft-collection (ultimo accesso 20/11/2021)

https://www.doppiozero.com/materiali/un-live-dei-corpi-distanza (ultimo accesso $10 / 11 / 2021)$

https://www.hdblog.it/mobile/articoli/n540833/nft-mercato-miliardi-2021-analisi (ultimo accesso 20/11/2021)

https://www.rollingstone.it/musica/guarda-levento-one-world-together-at-home/513109 (ultimo accesso 3/11/2021)

https://www.wired.it/play/musica/2021/03/04/blockchain-nfts-industria-musicalesostenibile (ultimo accesso 5/11/2021)

\section{Biografie degli autori/Authors' biographies}

Alfonso Amendola è professore associato di Sociologia dei processi culturali presso l'Università degli Studi di Salerno. Delegato del Rettore della Web Radio d'Ateneo. Coordina il laboratorio di progettazione culturale "Open Class". Si occupa di sociologia delle arti, dello spettacolo e della letteratura con particolare attenzione verso i modelli d'avanguardia, la digital society e i consumi di massa (temi su cui ha pubblicato numerosi saggi e lavori monografici). Redattore di riviste internazionali, editorialista del quotidiano "II Mattino" e project manager di eventi culturali.

Alfonso Amendola is associate professor of Sociology of Cultural Processes at the University of Salerno. $\mathrm{He}$ is the Rector's delegate for the University Web Radio. He coordinates the "Open Class" cultural design workshop. He deals with the sociology of the arts, entertainment, and literature, with particular 
emphasis on avant-garde models, the digital society, and mass consumption (topics on which he has published numerous essays and monographic works). He is the editor of international magazines, a columnist for the daily newspaper II MATTINO and a project manager for cultural events.

Michelle Grillo è dottoranda in Medium e Medialità presso l'Università eCampus. Cultrice della materia presso l'Università degli Studi di Salerno, collabora con le cattedre di Sociologia dell'immaginario tecnologico e Media classici e media digitali. Si occupa di social network society, consumi digitali, sociologia della musica e sociologia della letteratura. Tra i suoi recenti lavori un saggio dal titolo Vetrina. Il ritratto di Dorian Gray, la morte, la fotografia e la costruzione del sé attraverso il selfie, all'interno dell'antologia 10 Keywords, a cura di Alfonso Amendola e Mario Tirino edito Francesco D’Amato Editore. Ha curato la bibliografia, sitografia e videografia del saggio Fenomenologia dei Joy Division, a cura di Alfonso Amendola e Linda Barone (uscita prevista luglio 2021). Dal 2021 è membro del comitato redazionale della collana della casa editrice Rogas, La sensibilità vitale, specializzata in Scienze dei media e Sociologia della cultura.

Michelle Grillo is a PhD student in Medium and Mediality at the eCampus University. She collaborates with the chairs of Sociology of the Technological Imaginary and Classical and Digital Media at the University of Salerno. She has been researching and working on social network society, digital consumption, sociology of music and sociology of literature. Her recent works include an essay entitled Vetrina. II ritratto di Dorian Gray, la morte, la fotografia e la costruzione del sé attraverso il selfie (The portrait of Dorian Gray, death, photography and the construction of the self through selfies) in the anthology 10 Keywords, edited by Alfonso Amendola and Mario Tirino and published by Francesco D'Amato Editore. She edited the bibliography, sitography, and videography of the essay Fenomenologia dei JoyDivision, edited by Alfonso Amendola and Linda Barone. Since 2021 she has been a member of the editorial board of the Rogas publishing house series, which specializes in Media Science and Sociology of Culture. 